\title{
Three-dimensional preoperative planning in the weight-bearing state: validation and clinical evaluation
}

\author{
Tabitha Roth ${ }^{1,2^{*}} \mathbb{0}$, Fabio Carrillo², Matthias Wieczorek ${ }^{3}$, Giulia Ceschi ${ }^{2}$, Hooman Esfandiari ${ }^{2}$, Reto Sutter ${ }^{4}$, \\ Lazaros Vlachopoulos ${ }^{5}$, Wolfgang Wein ${ }^{3}$, Sandro F. Fucentese ${ }^{5}$ and Philipp Fürnstahl ${ }^{2}$
}

\begin{abstract}
Objectives: 3D preoperative planning of lower limb osteotomies has become increasingly important in light of modern surgical technologies. However, 3D models are usually reconstructed from Computed Tomography data acquired in a non-weight-bearing posture and thus neglecting the positional variations introduced by weight-bearing. We developed a registration and planning pipeline that allows for 3D preoperative planning and subsequent 3D assessment of anatomical deformities in weight-bearing conditions.
\end{abstract}

Methods: An intensity-based algorithm was used to register CT scans with long-leg standing radiographs and subsequently transform patient-specific 3D models into a weight-bearing state. 3D measurement methods for the mechanical axis as well as the joint line convergence angle were developed. The pipeline was validated using a leg phantom. Furthermore, we evaluated our methods clinically by applying it to the radiological data from 59 patients.

Results: The registration accuracy was evaluated in 3D and showed a maximum translational and rotational error of $1.1 \mathrm{~mm}$ (mediolateral direction) and $1.2^{\circ}$ (superior-inferior axis). Clinical evaluation proved feasibility on real patient data and resulted in significant differences for 3D measurements when the effects of weight-bearing were considered. Mean differences were $2.1 \pm 1.7^{\circ}$ and $2.0 \pm 1.6^{\circ}$ for the mechanical axis and the joint line convergence angle, respectively. 37.3 and $40.7 \%$ of the patients had differences of $2^{\circ}$ or more in the mechanical axis or joint line convergence angle between weight-bearing and non-weight-bearing states.

Conclusions: Our presented approach provides a clinically feasible approach to preoperatively fuse 2D weight-bearing and 3D non-weight-bearing data in order to optimize the surgical correction.

Keywords: 3D preoperative planning, Weight-bearing, Osteotomy, 2D-3D registration, 3D measurement methods

\section{Key points}

- Preoperative planning of orthopaedic surgeries is increasingly performed using CT-reconstructed 3D models.

\footnotetext{
*Correspondence: tabitha.roth@balgrist.ch

${ }^{1}$ Institute for Biomechanics, ETH Zurich, Leopold-Ruzicka-Weg 4,

8093 Zurich, Switzerland

Full list of author information is available at the end of the article
}

- CTs are acquired in lying position and do not contain weight-bearing information.

- We suggest registering CTs with standing radiographs to obtain weight-bearing 3D models.

- Registration of 59 patients showed significant differences between non-weight-bearing and weight-bearing positions.

- 2D-3D registration is a clinically feasible method to obtain weight-bearing 3D models. 


\section{Background}

Malalignment of the lower limb often causes a shift of the load-bearing axis away from the center of the knee, leading to an imbalance in load distribution within the knee joint. Various studies have demonstrated correlations between the aforementioned malalignment and the development of knee osteoarthritis (OA) [1, 2], including not only chondral damage but also alterations in bony microstructure of the underlying subchondral trabecular bone $[3,4]$. While progressed degeneration requires surgical treatment through unicompartmental or total knee replacement (TKR) [5], a joint-preserving realignment surgery achieved through a corrective osteotomy is the benchmark in younger patients with unilateral OA [6]. In a corrective osteotomy intervention, the pathologically deformed bones are cut, realigned and subsequently fixed with an orthopedic implant, transferring the load from the pathological to the healthy compartment of the knee. Precise postoperative alignment to a neutral weight-bearing axis or even mild over-corrections have been found to result in improved functional scores [7]. To achieve this, accurate preoperative planning and radiographic measurements are crucial.

Traditionally, realignment surgeries were planned in two dimensions (2D) using long-leg standing radiographs in order to realign the loaded region and achieve an optimal joint alignment, which is relevant to counteract OA development $[8,9]$. Technical advances of the recent years gave rise to modern computer-assisted surgery methods that are based on 3D models of the patient anatomy $[10,11]$. These 3D models are generated from CT scans, which are acquired in a supine, non-weightbearing position. By using these models, potential inaccuracies can occur in the preoperative plan that are attributed to the non-weight bearing position of the subject during the image acquisition, but one would need to make this compromise to gain the ability of planning in $3 \mathrm{D}$. We have recently shown that the relevant radiological metrics in the context of preoperative planning, such as the mechanical axis (MA) and the joint line convergence angle (JLCA), are significantly different between $2 \mathrm{D}$ weight-bearing and $2 \mathrm{D}$ non-weight-bearing as well as between 2D non-weight-bearing and 3D non-weightbearing conditions [12].

In the current clinical practice, there are no validated computer methods that allow for 3D preoperative planning of corrective osteotomies under weight-bearing conditions. We suggest to address this gap by implementing an algorithm to register the 3D non-weight-bearing imaging data to the $2 \mathrm{D}$ weight-bearing counterparts. In orthopedics, 2D-3D registration has already been widely used in various applications, such as in kinematic analyses, image-guided interventions or postoperative estimation of implant position [13-20]. For example, algorithms have been developed to accurately estimate the 3D pose of prosthesis components after TKR from single fluoroscopy images. These algorithms, which are from the feature-based registration family, solve the registration problem by iteratively projecting the contours of the $3 \mathrm{D}$ surface model onto the $2 \mathrm{D}$ image and minimizing the discrepancies between the projected contours and the actual 2D contours $[14,15]$.

Similarly, 2D-3D registration algorithms relying on single-plane fluoroscopy images were applied to study tibiofemoral kinematics during static and dynamic weight-bearing postures. While some groups also used feature-based approaches [16, 17], more recent publications describe so-called intensity-based algorithms [18-20]. In such techniques, synthetic 2D images called Digitally Reconstructed Radiograph (DRRs) are first obtained from the volumetric CT scans placed in an initial position. Later, the CT scan is iteratively transformed into a new position from which new DRRs are generated. A similarity metric is calculated at each point between the DRR and the actual 2D radiograph and the global optima transformation is found at a point resulting in the highest similarity. However, the out-of-plane registration error in all of these studies were shown to be too large for the purpose of 3D preoperative planning. Additionally, these approaches only focus on the knee joint itself and do not consider the alignment of the entire leg.

For the purpose of preoperative planning of corrective osteotomies, the translation and rotation in the coronal plane is of particular interest. Using biplanar long-leg standing radiographs as a registration target is therefore a reasonable choice to maximize registration accuracy also in the frontal plane. Fuji et al. [21] used one X-ray source in combination with a rotational table, which was positioned at $0^{\circ}$ and $60^{\circ}$ relative to the optical axis of the $\mathrm{X}$-ray source. Calibration for the two positions was performed manually beforehand.

Specialized centers are increasingly replacing conventional radiographs with the EOS imaging system (EOS imaging system, EOS, Paris, France), a pre-calibrated imaging modality capable of providing biplanar low-dose standing long-leg radiographs. This system utilizes two synchronized $\mathrm{x}$-ray beams, perpendicular to one another, which scan the subject from top to bottom in a vertical movement. The EOS device therefore has a different imaging geometry than conventional projective radiographs [22].

Given the clinical evidence that malalignment along with its associated change in load distribution are independent risk factors for OA progression [23], the goal of our study was to develop a pipeline that allows for $3 \mathrm{D}$ preoperative planning under weight-bearing 
conditions. To this end, we leveraged a $2 \mathrm{D}-3 \mathrm{D}$ registration algorithm with biplanar EOS 2D images as the registration target to transform the 3D models from a non-weight-bearing to a weight-bearing posture. Thereafter, we validated the pipeline in terms of registration accuracy and evaluated its clinical feasibility in a retrospective study.

\section{Methods}

The method section is organized in three parts. In the first part, we describe our developed registration and measurement pipelines capable of transferring 3D models into a weight-bearing posture and assess the MA as well as the JLCA in 3D (Fig. 1). As the input to our model, we use CT scans of the hip, knee and ankle joints as well as biplanar EOS images of the entire leg. As the output, the user receives 3D MA and JLCA measurements assessed in a weight-bearing posture.

In the second part, the accuracy of the registration algorithm is calculated in the form of a quantitative validation. Lastly, the clinical feasibility of our approach was evaluated retrospectively by applying our pipeline to a larger radiological dataset of patients who underwent corrective osteotomy of the leg.

\section{D model generation module}

Segmentation of the CT data was performed using a commercial segmentation software (Mimics Medical 19.0, Materialise NV, Leuven, Belgium). 3D models were generated using the Marching Cube algorithm [24] and were represented in the form of triangular surface meshes (stereolithographic models; STL) as described by [25]. The femoral and tibial segments were then transformed to a weight-bearing state by applying the transformation matrices obtained from the registration module (described below). The transformation of the 3D segments was done in Matlab (Matlab 2019a, The MathWorks Inc., Natick, MA, USA).

\section{Registration module}

The registration algorithm finds the optimal position by comparing intensity values between the EOS radiographs and DRRs [27], generated from CT and using the same geometry as the EOS system. Contrary to a standard $\mathrm{C}$-arm $\mathrm{x}$-ray system, the EOS system uses a fan-beam scan approach in which only the horizontal axes of the images ( $\mathrm{x}$ and $\mathrm{y}$ ) are affected by projective effects, introducing magnification in this direction (Fig. 2). The vertical z-axis of the image on the other hand directly relates, without magnification, to the $\mathrm{z}$-axis in the world coordinate system. In order to mitigate the magnification

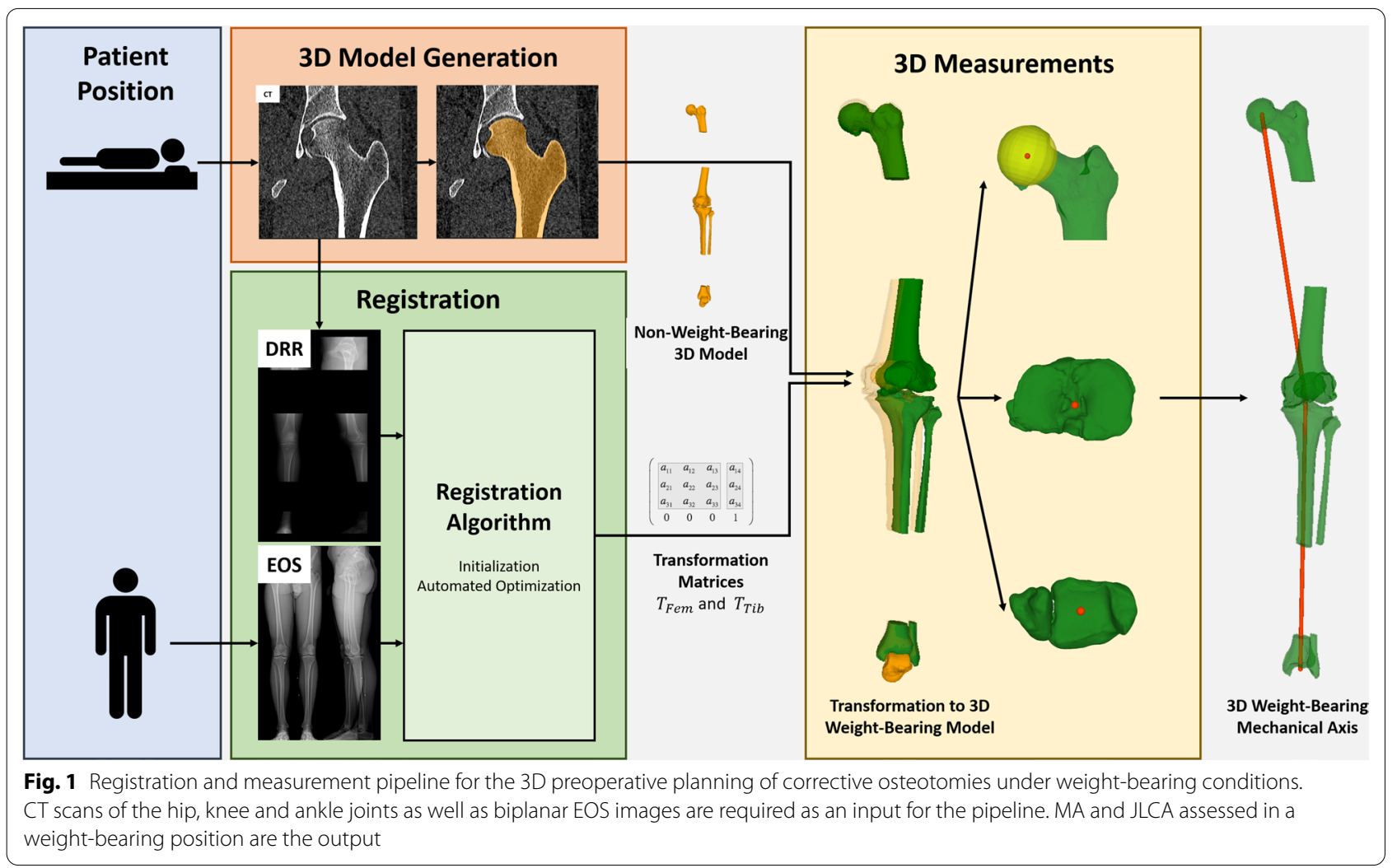




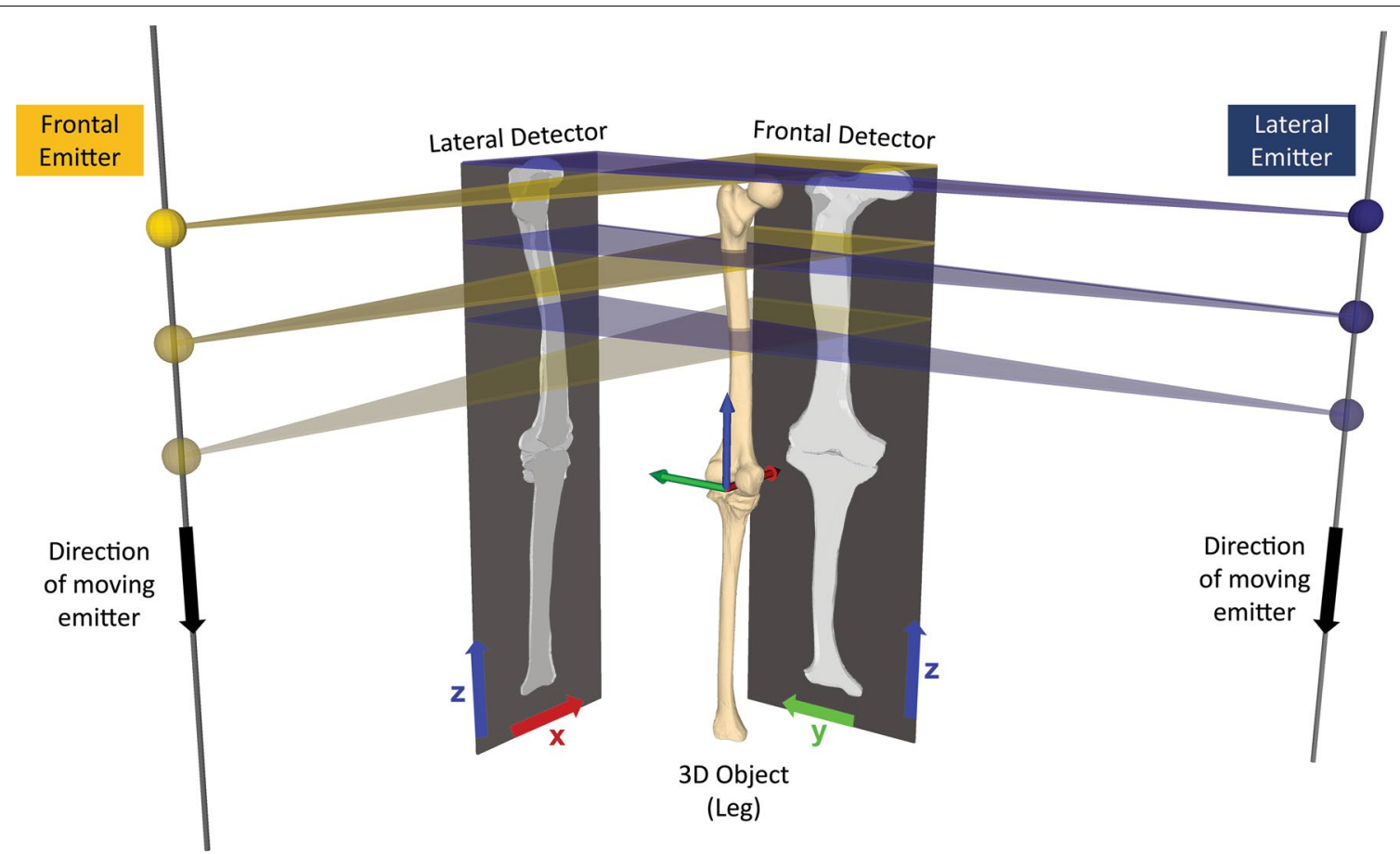

Fig. 2 Geometry of the EOS System. The EOS imaging systems produces biplanar long-leg standing X-rays by using a fan-beam scan approach, in which only the horizontal axis of the image is affected by projective effects. Frontal (in yellow) and lateral (in blue) emitters are depicted as spherical sources. The emitters move synchronously along the z-axis (shown in green). The vertical y-axes of the lateral and frontal detectors directly relate, without magnification, to the z-axis in the EOS coordinate system

effect on the horizontal axes, the EOS system rescales the images such that the they are virtually moved to a detector which is placed at the isocenter of the system, i.e. within the patient. All information required to reconstruct the EOS geometry were retrieved from the DICOM tags of the EOS images. A detailed description of the geometry can also be found in [22].

The EOS images as well as the CT served as the input for the registration algorithm, which was used to individually register femur and tibia to the EOS images. Two pre-processing steps were required for the algorithm: First, the registration was initialized based on three userselected annotations. An arrow connecting the same two anatomical landmarks was drawn in both the frontal and lateral EOS radiographs (2D) as well as the CT scan (3D) (Fig. 3). For example, the three arrows for the tibia were drawn from the distal end of the tibia to the center of the tibial plateau in all three images. Based on the aforementioned EOS geometry, the 2D image coordinates of the start and end points of the EOS arrow annotations (frontal and lateral) were subsequently triangulated, i.e. the 3D coordinates of the start and end point were reconstructed from their frontal and lateral 2D projections. The resulting 3D coordinates in the EOS space were used together with the $3 \mathrm{D}$ coordinates of the arrow annotation in the
CT space (also connecting the condyle midpoint with the center of the femoral head) to apply an initial transformation on the entire CT volume so that its projection on the EOS planes (DRRs) were roughly congruent with the EOS images (i.e. to initialize the 2D-3D registration process).

In the second pre-processing phase, the user had to draw a rectangular mask around the respective bone (femur or tibia) in both planes of the EOS in order to define the desired region of interest in which the registration was conducted.

Following the notion of intensity-based 2D-3D registration, our registration was achieved through an optimization routine, through which the pose of the CT scan was optimized with respect to a six degrees-of-freedom transformation (3 Euler angles and 3 translational parameters), resulting in a $4 \times 4$ transformation matrix $T$. After each optimization step, new DRRs were generated using the updated CT pose and subsequently compared with the corresponding EOS images. As the cost function, we use the sum of a variance-weighted localized normalized cross correlation (LNCC) similarity with a patch size of nine pixels between the simulated DRR and the corresponding EOS images [28]. The optimization executed using a bound optimization by quadratic approximation 


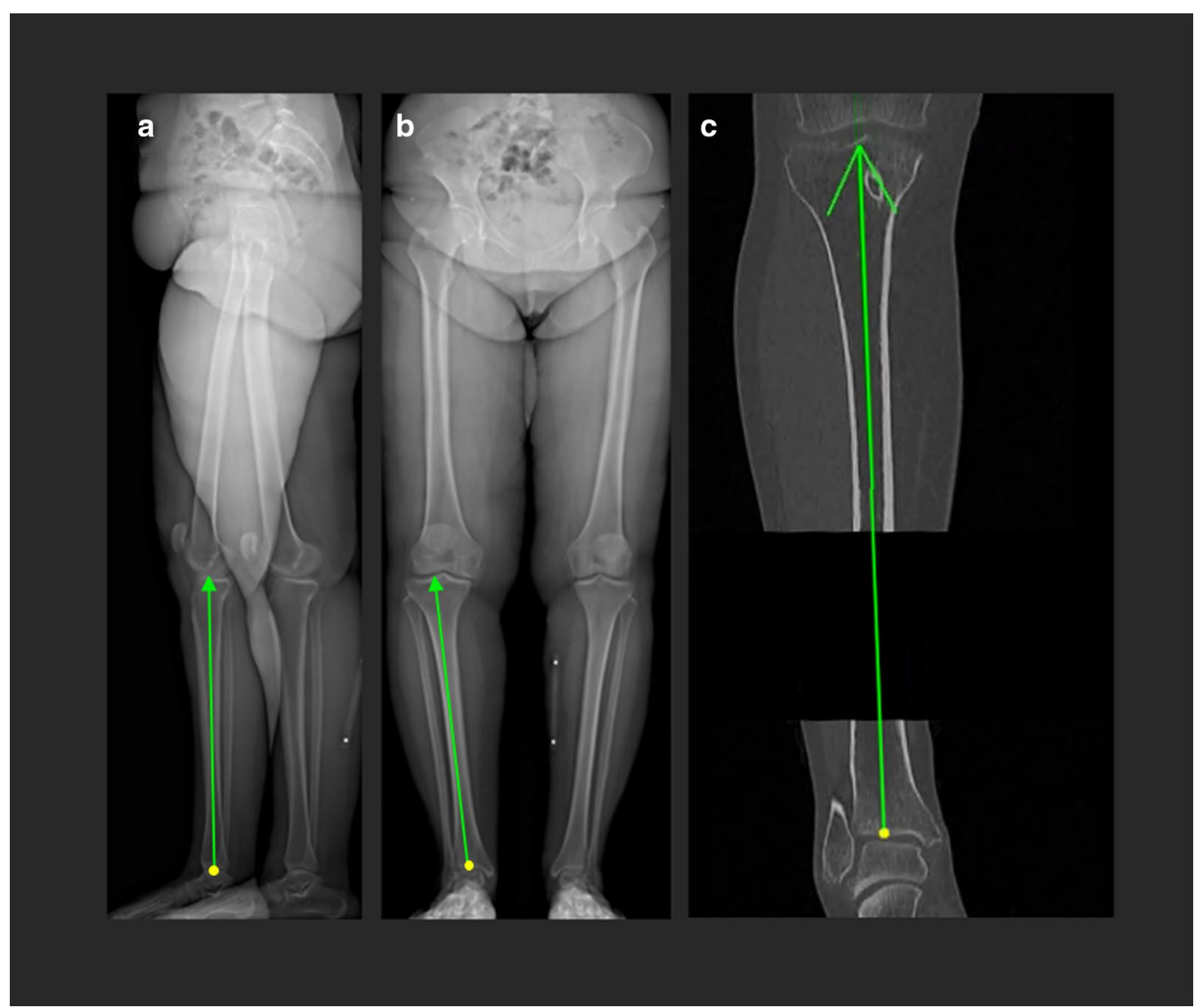

Fig. 3 Manual annotations to initialize the registration algorithm. Three user selected annotations are used to find the initial position between EOS and DRR. In this example, an arrow is drawn from the distal to the proximal end of the tibia in the two EOS images (a, b) as well as the CT scan (c)

(BOBYQA) algorithm [29] from the nlopt library that is relatively fast and does not require the calculation of cost function derivatives [30].

The output of the registration algorithm is in the form of a $4 \times 4$ transformation matrix, combining a $3 \times 3$ rotation matrix and a $1 \times 3$ translation vector, describing the relative transformation from the $3 \mathrm{D}$ coordinate system of the EOS to the coordinate system of the CT.

Registration is performed individually for the femoral and the tibial segments, resulting in the transformation matrices $T_{\mathrm{EOS}}^{\mathrm{CT}}(\mathrm{FEM})$ and $T_{\mathrm{EOS}}^{\mathrm{CT}}$ (TIB), respectively.

The registration algorithm was implemented within the ImFusion Suite software environment (ImFusion GmbH, Munich, Germany).

\section{D measurement module}

Once the registration is completed, the non-weight-bearing 3D models of femur and tibia were transformed using $T_{\mathrm{EOS}}^{\mathrm{CT}}(\mathrm{FEM})$ and $T_{\mathrm{EOS}}^{\mathrm{CT}}(\mathrm{TIB})$, respectively, and used as a representative of the weight-bearing posture. To this end, a 3D measurements module was developed that took the registered 3D models as input in order to calculate MA and JLCA under weight-bearing conditions.

First, the hip, knee and ankle joint centers were determined as described in [11]. The hip joint center (HJC) was defined as the center of a sphere, fitted to the userselected points of the femoral head using least-square regression [31] (Fig. 4a). The knee joint center (KJC) was defined as the midpoint between the intercondylar eminences of the tibial plateau (Fig. 4b). Thereafter, the ankle joint center (AJC) was determined as the center of all points belonging to the distal articular surfaces of the femoral and the tibial segments. These points on the articular surface were found by calculating the closestpoint distance to the talus model and considering only points below a user-defined distance threshold. The threshold is visually determined for each case until the entire articular surface is selected (Fig. 4c).

Using these landmarks, the 3D leg models were reoriented in a right-handed anatomical coordinate system. The mechanical leg axis (HJC to AJC) was aligned perpendicular to the axial plane (superior direction along the positive $y$-axis), and subsequently rotated along this 


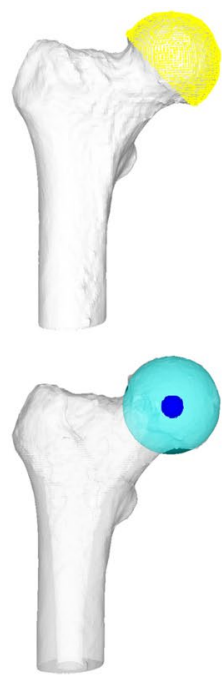

a
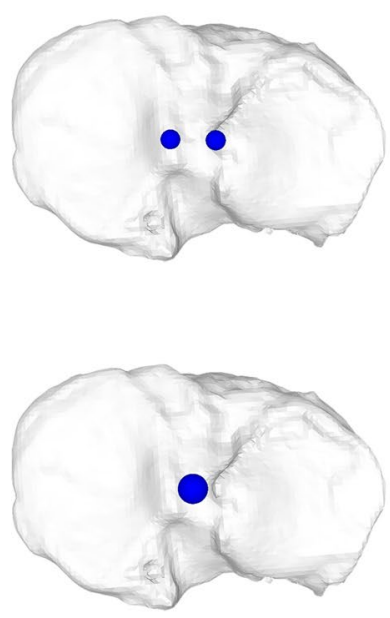

b

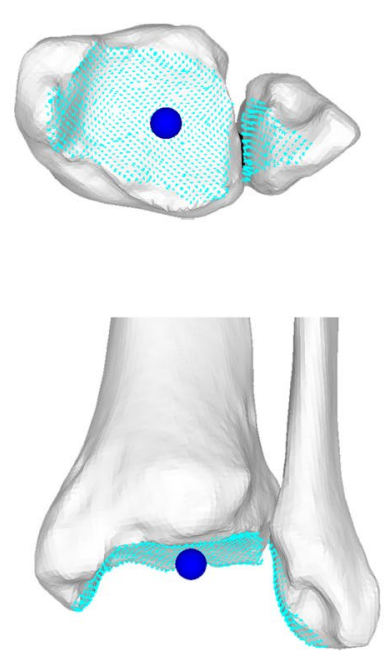

C

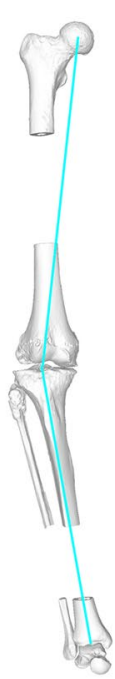

d

Fig. 4 Calculation of the mechanical axis in 3D. a The HJC is defined as the center of a sphere fitted to the femoral head. $\mathbf{b}$ The KJC is located in between the intercondylar eminences on the tibial plateau. c The AJC is defined as the center of all points of the distal tibial and fibular articular surfaces

axis until the anterior surface of the patella was parallel to the frontal plane (anterior direction along the positive $\mathrm{z}$-axis). The MA and JLCA were assessed in 3D using the methods as follows. Varus MA and laterally opened JLCA were denoted as positive, valgus MA and medially opened JLCA as negative.

The MA was assessed as described in [11] based on the hip joint, knee joint and ankle joint centers (Fig. 4d). Given the centers, the MA was calculated as the angle between a line connecting the $\mathrm{HJC}$ and the $\mathrm{KJC}$ and a second line between the $\mathrm{KJC}$ and $\mathrm{AJC}$, projected to the frontal plane.

To measure the JLCA, the longitudinal axis of the femur was determined by applying principal component analysis on all points of the bone model [31]. The distal femoral epiphysis was then isolated by cutting the femur right above the femoral epicondyles. A k-means algorithm [32], initialized as in [33], was used to automatically divide the epiphysis into the two condyles. The three most distal points along the longitudinal axis were found for each condyle cluster (Fig. 5a), and the femoral condyle tangent (FCT) was drawn by connecting their means.

The tibial condyle tangent (TCT) was defined as the frontal projection of the tibial plateau plane. The tibial plateau plane was defined using a least squares approach [31], minimizing the distance to eight userselected points, arranged as rhomboids on the tibial plateaus (Fig. 5b). Finally, the JLCA was defined as the angle between the frontal projection of the FCT and the TCT (Fig. 5c). These measurements were performed using our in-house planning software CASPA (CASPA 5.32, University Hospital Balgrist, Zurich, Switzerland) and Matlab (Matlab 2019a, The MathWorks Inc., Natick, MA, USA).

\section{Validation}

The accuracy of the registration module was assessed using a leg phantom (full leg 1132-48, Sawbones, Vashon Island, USA). The phantom was rigidly mounted on a wooden frame and each bone was equipped with a set of six radiopaque spherical markers of $3 \mathrm{~mm}$ diameter, visible both in the CT volume and the EOS radiographs. The validation medium was scanned using a CT scanner with a slice thickness of $0.6 \mathrm{~mm}$ and an in-plane pixel spacing of $0.26 \mathrm{~mm}$ (Somatom Definition AS Siemens Healthcare, Erlangen, Germany). Subsequently, two orthogonal long-leg standing radiographs were acquired using the EOS system.

To obtain the ground truth transformation for the validation setup, we first calculated the 3D coordinates of each marker in the CT scan's coordinate system $\mathrm{CS}_{\mathrm{CT}}$ by manually segmenting the $\mathrm{CT}$ scan to acquire a 3D model of the marker. The centers of the resulting 3D markers were found using a sphere-fitting algorithm [34].

Later, the 3D coordinates $\left(P_{x}, P_{y}, P_{z}\right)$ of the center of each marker were determined with respect to the $3 \mathrm{D}$ EOS coordinate frame $C S_{\mathrm{EOS}}$ based on their projections in the frontal and lateral EOS images using the following pair of linear equations: 


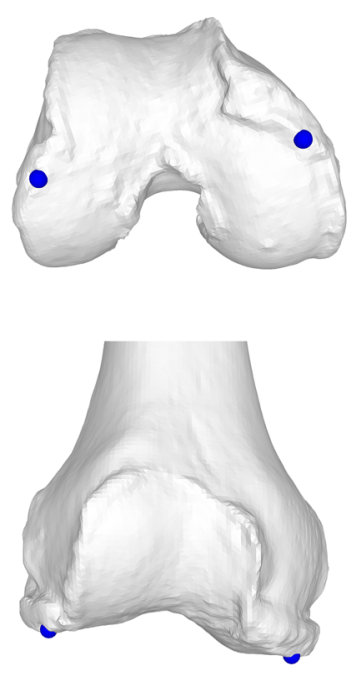

a

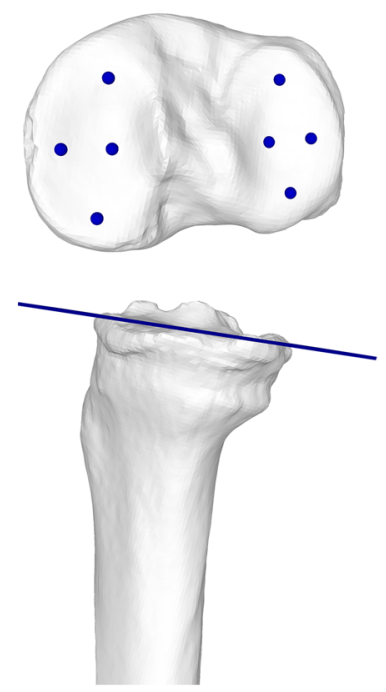

b

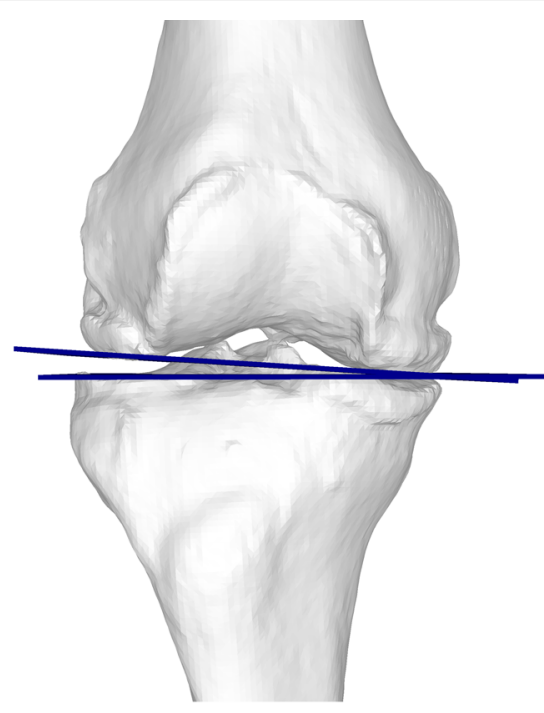

C

Fig. 5 Calculation of the joint line convergence angle in 3D. a The FCT is defined by the two most distal points, one on each condyle. b The TCT is found by defining the tibial plateau plane using user selected points on the plateau. C Both the FCT and the tibial plateau plane are projected to the frontal plane to find the JLCA

$$
\begin{aligned}
& \left(\begin{array}{cc}
-h_{f} & f_{f} \\
f_{l} & -h_{l}
\end{array}\right)\left(\begin{array}{c}
P_{x} \\
P_{y}
\end{array}\right)=\left(\begin{array}{ll}
f_{f} & h_{f} \\
f_{l} & h_{l}
\end{array}\right) \\
& P_{z}=z_{0}-\lambda_{z}\left(\frac{\nu_{l}+v_{f}}{2}\right)
\end{aligned}
$$

where $h_{f}$ and $h_{l}$ correspond to the horizontal position of the landmark in image (pixel) space in the frontal and lateral planes, respectively. $f_{f}=918 \mathrm{~mm}$ and $f_{l}=918 \mathrm{~mm}$ correspond to the distance of the frontal and lateral emitter to the EOS isocenter. Additionally, $z_{0}=477.5 \mathrm{~mm}$ is the initial height of the emitter, $\lambda_{z}=0.179363 \mathrm{~mm}$ is the pixel pitch of the image in the vertical direction and $v_{l}, v_{f}$ are the vertical position of the landmark in image (pixel) space in the lateral and frontal planes. More details regarding the EOS reconstruction geometry can be found in [22].

Once the 3D EOS coordinates of each marker were determined for each bone, we applied an iterative closest point (ICP) registration algorithm [35] to register the set of twelve corresponding 3D marker coordinates, to find the ground truth transformation matrix between the $3 \mathrm{D} C \mathrm{CT}$ and the $3 \mathrm{D}$ EOS coordinate sets $\left(\mathrm{GT}_{\mathrm{EOS}}^{\mathrm{CT}}\right)$.

$\mathrm{CT}$ and EOS radiographs were then registered using the previously described registration module, individually for tibia and femur, resulting in the transformation matrices $T_{\mathrm{EOS}}^{\mathrm{CT}}(\mathrm{Fem}), T_{\mathrm{EOS}}^{\mathrm{CT}}(\mathrm{Tib})$.

Finally, in order to validate our registration method, the registration error was obtained as
$E_{\mathrm{Fem}}=T_{\mathrm{EOS}}^{\mathrm{CT}}(\mathrm{Fem})^{-1} * \mathrm{GT}_{\mathrm{EOS}}^{\mathrm{CT}}$ for the femur, and $E_{\mathrm{Tib}}=T_{\mathrm{EOS}}^{\mathrm{CT}}(\mathrm{Tib})^{-1} * G T_{\mathrm{EOS}}^{\mathrm{CT}}$ for the tibia.

Our right-handed coordinate systems were oriented with the $\mathrm{x}$-axis to the left, while the $\mathrm{y}$ - and $\mathrm{z}$-axes correspond to the posterior and superior directions, respectively.

\section{Clinical feasibility study on patient data}

For the clinical feasibility assessment, we included patients treated in our institution who underwent a corrective osteotomy procedure at either distal femur and/ or proximal tibia between January 2015 and May 2020. Patients with an incomplete radiological dataset were excluded, resulting in a total of 55 patients, of whom 4 were operated on both legs. The mean age of the patients at the time of surgery was $43.5 \pm 8.4$ years, with 29 left and 30 right leg operation, and a gender distribution of 15 females and 44 males.

A full radiological dataset consisted of the following:

- A biplanar standing long-leg radiograph (EOS imaging system, EOS, Paris, France)

- A CT scan (Philips Brilliance 64, Philips Healthcare, Best, The Netherlands, or Somatom Definition AS Siemens Healthcare, Erlangen, Germany) according to the MyOsteotomy CT protocol (Medacta SA, Switzerland). Thereby, the hip, knee and ankle joints were scanned while the midshaft regions were skipped in order to reduce radiation exposure. Slice 
thickness was $1 \mathrm{~mm}$ with a spacing of either 0.5 or $0.8 \mathrm{~mm}$, while in-plane resolution was either 0.2 or $0.4 \mathrm{~mm}$.

The 3D preoperative planning was then performed for all the 59 legs using our registration and measurement pipeline, resulting in transformation matrices for femur and tibia, respectively.

All registrations were performed by the same reader. Out of the 59 cases, 20 were randomly selected and registered by a second reader in order to assess inter-rater variability.

Asymptotic Wilcoxon tests were applied (IBM SPSS for Windows, version 26, Armonk, NY, USA) to analyze the differences of means between weight-bearing and nonweight-bearing values. Mean absolute differences (MAD) were calculated between weight-bearing and non-weightbearing states. The correlation between MADs of JLCA and MA were assessed using the Pearson correlation coefficient. The interrater reliability was calculated with the intra-class correlation coefficient (ICC, two-way random model of single measures). Tests were evaluated using a significance level of $p \leq 0.05$.

\section{Results}

\section{Validation}

The translation error between ground truth transformation $\left(\mathrm{GT}_{\mathrm{EOS}}^{\mathrm{CT}}\right)$ and implemented registration $\left(T_{\mathrm{EOS}}^{\mathrm{CT}}(\mathrm{Tib})\right.$ and $\left.T_{\mathrm{EOS}}^{\mathrm{CT}}(\mathrm{Fem})\right)$ was $1.1,0.3$ and $-0.1 \mathrm{~mm}$ and $0.0,0.5$ and $-0.4 \mathrm{~mm}$ for femur and tibia in $\mathrm{x}, \mathrm{y}$ and $\mathrm{z}$ direction, respectively. This results in Euclidean distances of $1.1 \mathrm{~mm}$ and $0.6 \mathrm{~mm}$ for femur and tibia. The rotation errors were estimated to be $0.0^{\circ}, 0.1^{\circ}$ and $1.2^{\circ}$ for the femur and $0.0^{\circ}$, $-0.2^{\circ}$ and $1.1^{\circ}$ for the tibia around $x-, y$ - and $z$-axes.

\section{Evaluation of clinical feasibility}

The registration and measurement pipeline could be successfully applied for all patients.

Mean absolute difference between weight-bearing and non-weight-bearing in $3 \mathrm{D}$ was $2.1 \pm 1.7^{\circ}$ (range $0^{\circ}-6.2^{\circ}$ ) for the MA. MA was statistically significantly different between weight-bearing and non-weight-bearing states (means 5.4 vs. $6.1^{\circ}$ for non-weight-bearing vs. weightbearing, $\mathrm{z}=-2.26, p=0.02)$. 22 out of 59 patients $(37.3 \%)$ had a difference of $2^{\circ}$ or more.

Mean absolute difference between weight-bearing and non-weight-bearing JLCA was $2.0 \pm 1.6^{\circ}$ (range $\left.0.1^{\circ}-7.5^{\circ}\right)$. JLCA was statistically significantly different between weight-bearing and non-weight-bearing (means 3.6 vs. $4.9^{\circ}$ for non-weight-bearing vs. weight-bearing, $\mathrm{z}=-3.89, p<0.001) .24$ out of 59 patients $(40.7 \%)$ had a difference of $2^{\circ}$ or more (Fig. 6).
The correlation coefficient for MADs of JLCA and MA was $0.752(p<0.001)$.

The ICC for interrater reliability was 0.961 (95\% CI $0.902-0.984, p<0.001)$.

\section{Discussion}

This study aimed at the validation and clinical evaluation of a registration and measurement pipeline for enabling the $3 \mathrm{D}$ preoperative planning of corrective osteotomies in a weight-bearing state. $3 \mathrm{D}$ preoperative surgery planning has become increasingly important, and we have therefore recently developed and validated 3D measurement methods to assess lower limb anatomical deformities [12]. In line with our results, it has been shown that deformities are significantly underestimated when assessed on CT-based 3D models [36], which will likely lead to under-correction during surgery. In this case, the load is not transferred to the contralateral compartment but remains on the osteoarthritic side. Studies have demonstrated that under-correction is significantly correlated with high failure rates [37] and poor clinical outcome when compared to accurate correction or a slight overcorrection [38]. On the other hand, adequate axis correction creates an environment that allows for partial or complete cartilage regeneration on the femoral condyles and tibial plateau $[39,40]$.

Our registration algorithm was successfully applied for all patients including cases with heavily deformed bones. The entire registration pipeline for one patient took approximately $10-15 \mathrm{~min}$, whereby the automated optimization only represents a few seconds. The remaining time is used to load the images and perform the manual initialization as well as the visual control of the registration result. In our experience, the automatic pose optimization is reliable and rarely requires correction by the user. If required, the user has the possibility to adjust the translational and rotational parameters of the current position and subsequently restart the automated optimization.

Our validation has shown a maximal error of $1.1 \mathrm{~mm}$ in translation (medial-lateral direction) and in $1.2^{\circ}$ rotation around the superior-inferior axis. Given the fact that the registration target only consists of frontal and lateral $\mathrm{X}$-rays, the slightly higher rotational errors around the vertical axis are understandable. Otherwise, the rotational errors of $0.0^{\circ}-0.2^{\circ}$ are comparable to what was reported in a related study $\left(0.1^{\circ}-0.3^{\circ}\right)$ [41]. Similarly, our translational errors were close to the results reported in the same study $(0.1-0.5 \mathrm{~mm})$ with the exception of the $1.1 \mathrm{~mm}$ we have found for the femur in $\mathrm{x}$-direction.

These errors are within a range that we consider to be clinically acceptable. However, the time of up to $15 \mathrm{~min}$ per patient might hinder the implementation of this 


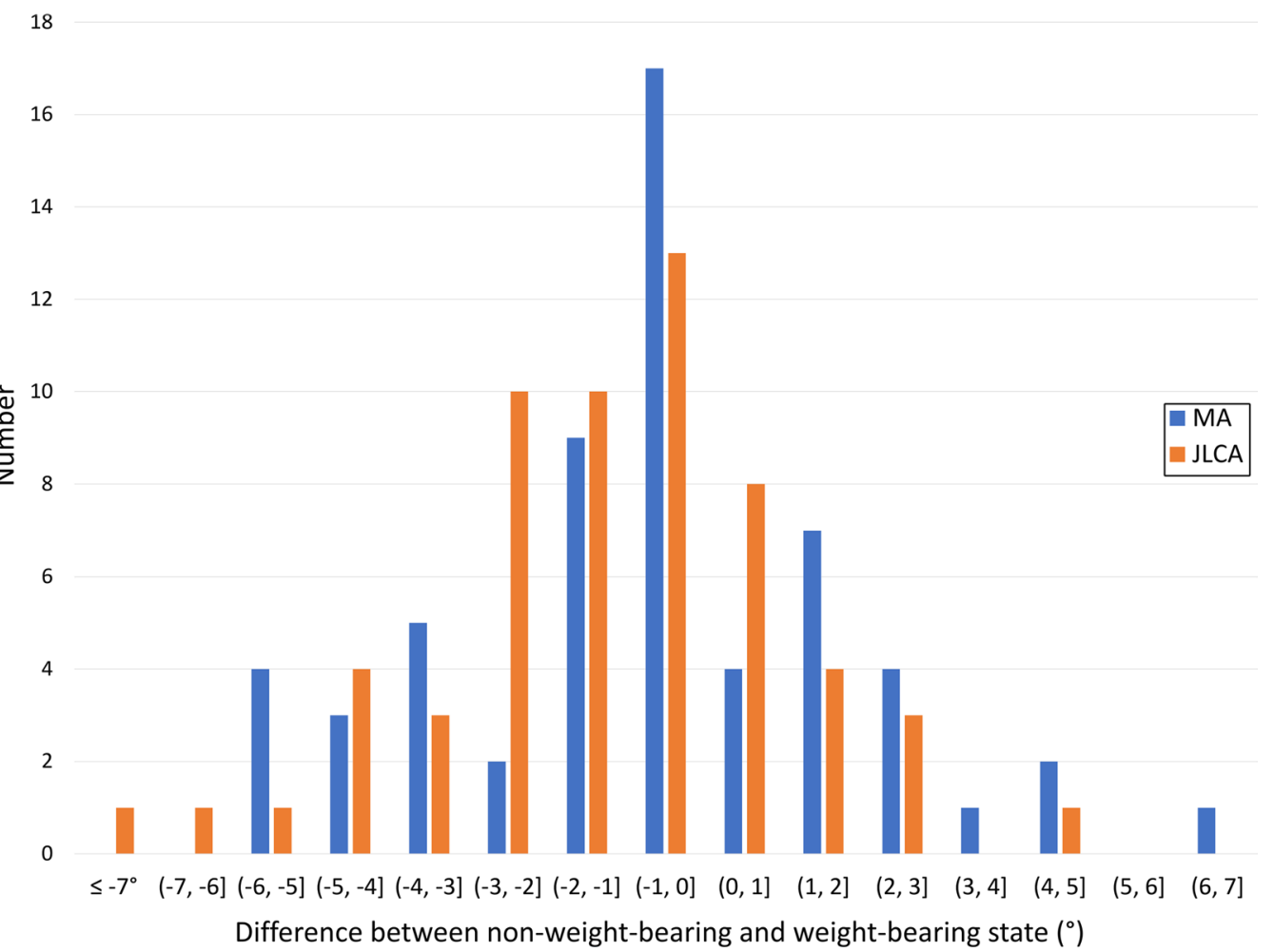

Fig. 6 Distribution of differences between non-weight-bearing and weight-bearing states for MA and JLCA. 37.3\% and 40.7\% of all patients have differences of $2^{\circ}$ or more between weight-bearing and non-weight-bearing positions for MA and JLCA, respectively

pipeline into the everyday clinical workflow. In our future work, we aim to implement an automated initialization by using deep learning networks for automated landmark detection. Given that studies have shown a detection accuracy of less than $1 \mathrm{~mm}$, it is reasonable to assume that this approach will help to find an initial overlapping between EOS and DRRs that is within the capture range of the algorithm. Furthermore, automated landmark detection will also enable the automated assessment of the anatomical deformity in 3D and therefore obviate the need for manual 3D measurements.

Our study is limited by several factors. First, our validation was only based on one sample. Furthermore, compared to the validation performed under laboratory conditions, additional challenges can be expected in a clinical setting that might influence the outcome of the registration. The registration accuracy in a clinical setting is strongly dependent on and limited by the quality of the EOS scans. Ideally, one leg is positioned slightly in front of the other in order to make both of them completely distinguishable in the sagittal scan. Notably more manual adjustment was required during the registration process in the cases with parallel leg position in the X-ray images. However, the patient should also equally distribute the weight between both legs, and knee flexion and the internal or external rotations should be kept minimal. This position can be challenging for patients with knee pain and can differ slightly from normal double-leg standing. Besides, the registration process is still partly dependent on the user and therefore not completely automated. However, since interrater reliability was very high, we assume that this does not affect the precision significantly.

\section{Conclusions}

A 2D-3D registration pipeline was developed and validated in order to transform non-weight-bearing 3D models of the lower limb into a weight-bearing state and therefore enables 3D preoperative planning under loaded conditions. Significant differences between weight-bearing and non-weight-bearing states in the clinical application have shown that it is important to consider the effects of weight-bearing in 3D preoperative planning. The presented registration and measuring pipeline is easy to use and could be implemented into the clinical workflow. 


\section{Abbreviations}

AJC: Ankle joint center; BOBYQA: Bound optimization by quadratic approximation; CT: Computer tomography; DRR: Digitally reconstructed radiograph; FCT: Femoral condyle tangent; HJC: Hip joint center; ICC: Intraclass correlation coefficient; ICP: Iterative closest point; JLCA: Joint line convergence angle; KJC: Knee joint center; LNCC: Localized normalized cross correlation; MA: Mechanical axis; MAD: Mean absolute difference; OA: Osteoarthritis; STL: Stereolithographic; TCT: Tibial condyle tangent; TKR: Total knee replacement.

\section{Acknowledgements}

Not applicable.

\section{Authors' contributions}

P.F. devised the project and main conceptual ideas and supervised the project in technical aspects. M.W. and W.W. developed and implemented the registration algorithm. F.C. and T.R. validated the algorithm with the help of H.E. G.C. and T.R. carried out the clinical data, T.R. performed the deformity measurements and statistical analyses. S.F. and L.V. supervised the project in the clinical aspects while R.S. was responsible for the radiological aspects. T.R. wrote the manuscript with the help of P.F., F.C., H.E. and M.W. All authors have read and approved the final submitted manuscript.

\section{Funding}

This study is part of the LEDECO project which was funded by the Swiss National Science Foundation (Grant Nr. 320030_182352 / 1).

\section{Availability of data and materials}

The dataset analyzed during the current study are not publicly available due to individual privacy reasons but are available from the corresponding author upon reasonable request.

\section{Declarations}

\section{Ethical approval and consent to participate}

The local ethical committee approved this study (Zurich Cantonal Ethics Commission, KEK 2018-02242) and all patients gave their written informed consent.

\section{Consent for publication}

Not applicable.

\section{Competing interests}

W.W. and M.W. are employed by ImFusion, which is a company developing a commercial software for medical image processing.

\section{Author details \\ ${ }^{1}$ Institute for Biomechanics, ETH Zurich, Leopold-Ruzicka-Weg 4, 8093 Zurich, Switzerland. ${ }^{2}$ Research in Orthopedic Computer Science (ROCS), Univer- sity Hospital Balgrist, University of Zurich, Balgrist Campus, Lengghalde 5, 8008 Zurich, Switzerland. ${ }^{3}$ ImFusion GmbH, Agnes-Pockels-Bogen 1, 80992 Munich, Germany. ${ }^{4}$ Department of Radiology, Balgrist University Hospital, University of Zurich, Forchstrasse 340, 8008 Zurich, Switzerland. ${ }^{5}$ Department of Orthopedics, Balgrist University Hospital, University of Zurich, Forchstrasse 340, 8008 Zurich, Switzerland.}

Received: 18 January 2021 Accepted: 24 March 2021

Published online: 07 April 2021

\section{References}

1. Sharma L, Song J, Dunlop D et al (2010) Varus and valgus alignment and incident and progressive knee osteoarthritis. Ann Rheum Dis 69(11):1940-1945

2. Sharma L, Chmiel JS, Almagor O et al (2013) The role of varus and valgus alignment in the initial development of knee cartilage damage by MRI: the MOST study. Ann Rheum Dis 72(2):235-240

3. Han X, Cui J, Xie K et al (2020) Association between knee alignment, osteoarthritis disease severity, and subchondral trabecular bone microarchitecture in patients with knee osteoarthritis: a cross-sectional study. Arthritis Res Ther 22(1):203
4. Renault JB, Carmona M, Tzioupis C et al (2020) Tibial subchondral trabecular bone micromechanical and microarchitectural properties are affected by alignment and osteoarthritis stage. Sci Rep 10(1):3975

5. Siman H, Kamath AF, Carrillo N et al (2017) Unicompartmental knee arthroplasty vs total knee arthroplasty for medial compartment arthritis in patients older than 75 years: comparable reoperation, revision, and complication rates. J Arthroplasty 32(6):1792-1797

6. Brouwer RW, van Raaij TM, Bierma-Zeinstra SMA et al (2007) Osteotomy for treating knee osteoarthritis. Cochrane Database Syst Rev 3:CD004019

7. Hohloch L, Kim S, Mehl J et al (2018) Customized post-operative alignment improves clinical outcome following medial open-wedge osteotomy. Knee Surg Sports Traumatol Arthrosc 26(9):2766-2773

8. Paley D, Herzenberg JE, Tetsworth K et al (1994) Deformity planning for frontal and sagittal plane corrective osteotomies. Orthop Clin North Am 25(3):425-465

9. Paley D (2002) Principles of deformity correction. Springer, Berlin

10. Fucentese SF, Meier P, Jud L et al (2020) Accuracy of 3D-planned patient specific instrumentation in high tibial open wedge valgisation osteotomy. J Exp Orthop 7(1):7

11. Fürnstahl P, Schweizer A, Graf A et al (2016) Surgical treatment of long-bone deformities: 3D preoperative planning and patient-specific instrumentation. In: Zheng G, Li S (eds) Computational radiology for orthopaedic intervensions. Springer, Cham

12. Jud L, Roth T, Fürnstahl $P$ et al (2020) The impact of limb loading and the measurement modality (2D versus 3D) on the measurement of the limb loading dependent lower extremity parameters. BMC Musculoskelet Disord 21(1):418

13. Markelj $P$, Tomaževič D, Likar B et al (2012) A review of 3D/2D registration methods for image-guided interventions. Med Image Anal 16(3):642-661

14. Zuffi S, Leardini A, Catani F et al (1999) A model-based method for the reconstruction of total knee replacement kinematics. IEEE Trans Med Imaging 18(10):981-991

15. Yamazaki T, Watanabe T, Nakajima Y et al (2004) Improvement of depth position in 2-D/3-D registration of knee implants using single-plane fluoroscopy. IEEE Trans Med Imaging 23(5):602-612

16. Dennis DA, Mahfouz MR, Komistek RD et al (2005) In vivo determination of normal and anterior cruciate ligament-deficient knee kinematics. J Biomech 38(2):241-253

17. Fregly BJ, Rahman HA, Banks SA (2005) Theoretical accuracy of modelbased shape matching for measuring natural knee kinematics with single-plane fluoroscopy. J Biomech Eng 127(4):692-699

18. Scarvell JM, Pickering MR, Smith PN (2010) New registration algorithm for determining 3D knee kinematics using CT and single-plane fluoroscopy with improved out-of-plane translation accuracy. J Orthop Res 28(3):334-340

19. Tsai TY, Lu T-W, Chen C-M et al (2010) A volumetric model-based 2D to $3 \mathrm{D}$ registration method for measuring kinematics of natural knees with single-plane fluoroscopy. Med Phys 37(3):1273-1284

20. Postolka B, Lista R, Thelen B et al (2020) Evaluation of an intensity-based algorithm for 2D/3D registration of natural knee videofluoroscopy data. Med Eng Phys 77:107-113

21. Fujii T, Sato T, Ariumi A et al (2020) A comparative study of weight-bearing and non-weight-bearing 3-dimensional lower extremity alignment in knee osteoarthritis. J Orthop Sci 25(5):874-879

22. Groisser B (2019) Geometry of the EOS (R) Radiographic Scanner. arXiv: 1904.06711 [eess.IV]

23. Tanamas S, Hanna FS, Cicuttini FM et al (2009) Does knee malalignment increase the risk of development and progression of knee osteoarthritis? A systematic review. Arthritis Rheum 61(4):459-467

24. Lorensen WE, Cline HE (1987) Marching cubes: a high resolution 3D surface construction algorithm. In: ACM siggraph computer graphics. ACM.

25. Roscoe L (1988) Stereolithography interface specification. America-3D Systems Inc, $\mathbf{2 7}$

26. Hartley R, Zisserman A (2004) Multiple view geometry in computer vision, 2nd edn. Cambridge University Press, Cambridge

27. Goitein M, Abrams M, Rowell D et al (1983) Multi-dimensional treatment planning: II Beam's eye-view, back projection, and projection through CT sections. Int J Radiat Oncol Biol Phys 9(6):789-797

28. LaRose D (2001) Iterative X-Ray/CT registration using accelerated volume rendering. In: Robotics Institute. Carnegie Mellon University, Pittsburgh, PA 
29. Powell MJD (2009) The BOBYQA algorithm for bound constrained optimization without derivatives. Report No. DAMTP 2009/NA06, CMS, University of Cambridge

30. Johnson SG The NLopt nonlinear-optimization package. http://github. com/stevengj/nlopt

31. Schneider P, Eberly DH (2002) Geometric tools for computer graphics. Elsevier, New York

32. Forgy E (1965) Cluster analysis of multivariate data: efficiency versus interpretability of classification. Biometrics 21(3):768-769

33. Arthur D, Vassilvitskii S (2007) k-means++: the advantages of careful seeding. In: SODA 2007: Proceedings of the eighteenth annual ACM-SIAM symposium on Dicrete algorithms, pp 1027-1035

34. Lukács G, Marshall A, Martin R (1997) Geometric least-squares fitting of spheres, cylinders, cones and tori. RECCAD, Deliverable Document 2 and 3, COPERNICUS project, (1068).

35. Besl PJ, McKay ND (1992) Method for registration of 3-D shapes. Sensor Fusion IV: Control Paradigms Data Struct 1611:586-607

36. Leon-Munoz VJ, López-López M, Martínez-Martínez F et al (2020) Comparison of weight-bearing full-length radiographs and computedtomography-scan-based three-dimensional models in the assessment of knee joint coronal alignment. Knee 27(2):543-551
37. Rudan JF, Simurda MA (1990) High tibial osteotomy A prospective clinical and roentgenographic review. Clin Orthop Relat Res 255:251-256

38. El-Azab HM, Morgenstern M, Ahrens P et al (2011) Limb alignment after open-wedge high tibial osteotomy and its effect on the clinical outcome. Orthopedics 34(10):e622-e628

39. Jung WH, Takeuchi R, Chun C-W et al (2014) Second-look arthroscopic assessment of cartilage regeneration after medial opening-wedge high tibial osteotomy. Arthroscopy 30(1):72-79

40. Kanamiya T, Naito M, Hara M et al (2002) The influences of biomechanical factors on cartilage regeneration after high tibial osteotomy for knees with medial compartment osteoarthritis: clinical and arthroscopic observations. Arthroscopy 18(7):725-729

41. Kobayashi K, Sakamoto M, Tanabe Y et al (2009) Automated image registration for assessing three-dimensional alignment of entire lower extremity and implant position using bi-plane radiography. J Biomech 42(16):2818-2822

\section{Publisher's Note}

Springer Nature remains neutral with regard to jurisdictional claims in published maps and institutional affiliations.

\section{Submit your manuscript to a SpringerOpen ${ }^{\circ}$ journal and benefit from:}

- Convenient online submission

- Rigorous peer review

- Open access: articles freely available online

- High visibility within the field

- Retaining the copyright to your article

Submit your next manuscript at $\gg$ springeropen.com 